\title{
STOE explaination for the "ether wind"
}

\author{
J.C. Hodge $\mathrm{e}^{1 *}$ \\ ${ }^{1}$ Retired, 477 Mincey Rd., Franklin, NC, 28734
}

\begin{abstract}
The Michelson-Morley, the Miller, and other experiments have detected an "ether wind" and have stirred a long-standing controversy. The Scalar Theory Of Everything (STOE) is applied to the measurements of the Miller experiment. The STOE's plenum is comparable to the ether. The result is the divergence of the plenum caused by the Sun and Moon and the measured direction of the "ether wind" are at an angle of $91^{\circ} \pm 8^{\circ}$ with a confidence of $1 \sigma$. This suggests that these experiments are measuring the degree of tilt relative to the plenum divergence. That is, the measurements are not detecting the changing light path that results in the real Lorentz matter contraction hypothesis, but rather the measurements are detecting the changing light speed and direction caused by the divergence of the plenum. The support for the Lorentz matter contraction hypothesis as a real contraction is removed.
\end{abstract}

keywords: ether, ether wind, Michelson-Morley experiment, the Miller experiment, Lorentz contraction

\section{INTRODUCTION}

The ether has been proposed to be a medium filling all space between particles that transmits waves and directs particles. The concept of ether recurs throughout history. More recently it has been called by many names such as quantum vacuum, gravitational ether, and spacetime.

The "luminiferous ether" was theorized for the propagation of light. It was modeled as a fluid. When a body such as the Earth moved through the ether, it should create a wind called the "ether wind" (Michelson \& Morley 1887). The Michelson-Morley Experiment (MMX) attempted to detect the "ether wind". Their result did detect a small positive value that was much less than expected and that has stirred a long-standing controversy. Special relativity assumes the speed of light is constant. The reality of the Lorentz material contraction in bodies derives from the model of a changing light path in the MMX.

Many others repeated their experiment. The Miller (1933) paper strongly supports some effect thought to be suggesting an ether. Miller calculated the

\footnotetext{
*E-mail: jchodge@frontier.com
} 
"ether drift" was directed along an axis with the southern apex at right ascension $4^{\mathrm{h}} 54^{\prime}$, declination $-76^{\circ} 33^{\prime}$ at $208 \mathrm{~km} / \mathrm{s}$.

This and other similar experiments by others have a positive result. There are notable issues with the results. The "velocity" and direction match no known relationship to the Earth's axial rotation, the Earth's revolution around the Sun, the Sun's motion around the Milky Way galaxy, or the cosmic microwave background. Further, most, if not all, concepts of the ether suggest the ether supports wave action that requires the ether to have inertia(Hodge 2016). Inertia suggests the ether has viscosity to slow heavenly body's movement. Because the planets and stars systems have not collapsed, this has not been observed. This results in a quandary about what did Miller detect?

The Scalar Theory Of Everything (STOE) suggests the Newton (1730) model as a starting concept. Newton speculated the wave in aether travels faster (not slower) than the corpuscles of light and directs the corpuscles' path (Query 17). Newton's analogy was of water waves. Newton seems to have suggested that particles are directed by the divergence of the aether and that particles produce the wave phenomena in the aether.

The STOE has a plenum (like an ether) that is one of the two primordial components of the universe from which everything emerges. Indeed, the need to support waves is the reason for the ether in models. The STOE suggests that the plenum supports wave action and, consequently, has the property of inertia (Hodge 2016). The speed of the plenum waves is much faster than light as (Newton 1730; van Flandern 1998) suggests. The faster speed was used to develop the successful simulation of diffraction experiments Hodge (2012). In addition, the simulation suggested the speed of photons changes with plenum density and can be directed by the plenum divergence $(\vec{\nabla} \rho)$.

This Paper suggests the "ether wind" experiments are detecting the divergence of the plenum cause by the Sun and Moon. The description of the model is in section 2. The Discussion and Conclusion are in section 3.

\section{The model and the calculation}

The STOE model of the "ether wind" data is that the plenum density and divergence changes the speed and direction of the photons. Therefore, the photons in the MMX and Miller experiment have detected such changes. Accordingly, the calculation of direction should be sensitive to the divergence of the plenum. This divergence is determined by the Sun and Moon and all other bodies in the universe according to the Universal Equation Hodge (2018). However, the contribution of all other bodies than the Sun and Moon is relatively constant within the distance of the Earths orbit. Therefore, the vector direction of the plenum depends on the Sun and Moon in the calculation.

The Horizons database ${ }^{1}$ was consulted to provide the position of the Sun and Moon on the four days at $00 \mathrm{Hrs}$ of Miller's observations. The vector potential

\footnotetext{
${ }^{1}$ https//ssd.jpl.nasa.gov/horizons.cgi on 19 April 2019
} 
of the Sun $\vec{V}_{\text {sun }}=-\frac{G M_{\text {sun }}}{r_{\text {sun }}^{2}} \vec{r}_{\text {sun }}$ and the Moon $\vec{V}_{\text {moon }}=-\frac{G M_{\text {moon }}}{r_{\text {moon }}} \vec{r}_{\text {moon }}$ where $G$ is the gravitation constant; $M_{\text {sun }}$ and $M_{\text {moon }}$ are the masses of Sun and Moon, respectively; and $\vec{r}_{\text {sun }}$ and $\vec{r}_{\text {moon }}$ are the distance from the Sun and Moon to Earth, respectively.

The total vector potential $\vec{V}=\vec{V}_{\text {sun }}+\vec{V}_{\text {sun }}$ for each day of Miller's data (4 days).

Using the unit vector in the direction determined by Miller's $\vec{M}$, the angle $\theta$ (day) between the $\vec{V}$ and $\vec{M}$ on each day is then calculated:

$$
\theta(\text { day })=\arccos \left(\frac{\vec{V} \bullet \vec{M}}{|\vec{V}||\vec{M}|}\right) .
$$

The four $\theta($ day $)$ values were then averaged to calculate the net angle $\theta$.

The result is $\theta=91^{\circ} \pm 8^{\circ}$ with a confidence at $1 \sigma$.

\section{Discussion and Conclusion}

The result suggests that the measured effect requires $\vec{\nabla} \rho \neq 0$. The observation tables in the experiments were held flat relative to Earth's gravity. Thus, Earths gravity was not included in the findings.

The STOE suggests the speed of waves in the plenum (gravity waves, also) is many orders of magnitude greater than the speed of light (van Flandern 1998). If a fluid has inertia, it should have viscosity. The successful simulation of diffraction of light included a term that represented the viscosity of matter in a fluid as proportional to the relative velocity (without turbulence). This term in the simulation had a very minor effect on the photons. This suggests the plenum viscosity has very small impact on heavenly bodies. In addition, the effect of the Source in spiral galaxies more than compensates for any friction loss experienced by the mass. Indeed, the rotation curves of spiral galaxies suggest matter is moving faster than the Keplerian model calculates. A possible effect to be noticed may occur in the cooling flow of elliptical galaxies.

The result is the divergence of the plenum caused by the Sun and Moon and the measured direction of the "ether wind" are at an angle of $91^{\circ} \pm 8^{\circ}$ with a confidence of $1 \sigma$. This suggests that these experiments are measuring the degree of tilt relative to the plenum divergence. That is, the measurement is not the changing light path that results in the real Lorentz matter contraction hypothesis, but rather the measurements are detecting the changing light speed and direction caused by the divergence of the plenum. The support for the Lorentz matter contraction hypothesis as a real contraction is removed. 


\section{REFERENCES}

\section{Acknowledgments}

\section{References}

Michelson, A.A. \& Morley, E.W., 1987, On the Relative Motion of the Earth and the Luminiforus Ether, American Journal of Science, 34 (203) p. 333-345.

Hodge, J.C., 2012, Photon diffraction and interference, IntellectualArchive, Vol.1, No. 3, P. 20,. http://intellectualarchive.com/?link=item\&id=597

Hodge, J.C., 2013, STOE Inertia, IntellectualArchive, Vol.6, No. 1, P. 1,. http://intellectualarchive.com/?link=item\&id=1814

Hodge, J.C., 2018, STOE replaces relativity and quantum mechanics, IntellectualArchive, Vol.7, No. 5, P. 9,. http://intellectualarchive.com/?link=item\&id=1979

Miller, D. C., 1933. The Ether-drift experiment and the determination of the absolute motion of the Earth, Rev. of Mod. Phys., 5 (2) p. 203-342.

Newton, I., Opticks based on the 1730 edition (Dover Publications, Inc., New York, 1952).

van Flandern, T., 1998. The speed of gravity? What the experiments say, Physics Letters A, 250, (1-3). 